\title{
38. INVESTIGATION OF THE MOTION OF PERIODIC COMET STEPHAN-OTERMA
}

\author{
M. YA. SHMAKOVA \\ Institute for Theoretical Astronomy, Leningrad, U.S.S.R.
}

\begin{abstract}
The orbit of $\mathbf{P} /$ Stephan-Oterma has been determined using 128 observations at the two apparitions (1867 and 1942) and taking into account perturbations by Venus to Neptune. The evolution of the comet's orbit has been studied over the interval 1660-2060.
\end{abstract}

The short-period comet Stephan-Oterma, with a revolution period of about 40 years, has been observed at two apparitions, in 1867 and 1942. The comet was discovered by Stephan on 1867 January 25 at Marseilles. Orbital elements were obtained by Becker (1891) from 65 post-perihelion observations and taking into account perturbations by Venus to Jupiter by Encke's method. The maximum residual was slightly larger than $1^{\prime \prime}$.

The comet was not observed at its next return to perihelion ( $T=1904$ May 23.931). It was rediscovered accidentally at the end of 1942 by Oterma, Whipple and Tevzadze. Dubyago (1943) determined the orbit from seven post-perihelion observations.

The aim of the present investigation has been to obtain a fairly reliable orbit from observations at the two apparitions. We first determined an orbit from the three observations 1942 Nov. 28, 1943 Jan. 6 and Feb. 8. This was then corrected using 74 observations in 1942-1943 and allowing for the perturbations by Venus to Neptune, with the result:

$$
\begin{aligned}
& \text { Epoch }=1942 \text { Oct. } 18.0 \\
& T=1942 \text { Dec. } 19.09137 \quad \varphi=59.41888 \\
& M_{0}=358.42444 \quad n=91 " 34666 \\
& \omega=358.27211 \quad q=1.59529 \mathrm{AU} \\
& \Omega=78.60846\} \quad 1950.0 \quad Q=21.34361 \mathrm{AU} \\
& i=17.90115 \quad P=38.84317 \mathrm{yr} .
\end{aligned}
$$

The mean error is 1.4 , with individual residuals rising to 3".5. We can see that the comet is a member of the Uranus family $(Q=21.3 \mathrm{AU})$.

Belyaev's programme was then used to integrate the comet's motion (by Cowell's method) from 1942 back to 1867 on the BESM-4 computer, and again taking into account perturbations by Venus to Neptune. This first integration was made using a variable step (5-40 days). The 1867 observations were precessed to equinox 1950.0 , most of the reference stars being reduced to the $\mathrm{FK}_{4}$ system. The $(\mathrm{O}-\mathrm{C})$ residuals in 1867 were in some cases as high as 2.4 . As an experiment, the osculating elements at two epochs, 1942 October 18 and 1867 October 6, were improved separately, using the Eckert-Brouwer method and the observations at both apparitions. From the 
results obtained one can see that it is more efficient to take for the improvement the elements for an epoch during the apparition which gives the largest residuals (in this case 1867). Since the 1867 residuals are so large the corresponding differential coefficients must be calculated with great accuracy. After the first improvement the 1867 residuals decreased by a factor of 30, but after integration forward the 1942-1943 residuals were found to be 3 orders higher. Three successive improvements were carried out in this manner, and the maximum residual decreased to $0: 7$.

In order to converge on a satisfactory result more rapidly, we considered next the effect of a change in the comet's mean motion. We applied a certain increment $(\Delta n=0.000003=0.011)$ to the osculating mean daily motion $\left(n_{0}\right)$ in 1867 . This had a negligible effect on the 1867 residuals (which were less than $2^{\prime \prime}$ ). Integration of this new orbit forward gave quite different residuals in 1942-1943, and by comparison with the residuals for the initial orbit we established that a correction $\Delta n_{1}=$ +0.00000032 was required. Numerical integration with a new value of the mean daily motion $\left(n_{1}=n_{0}+\Delta n\right)$ yielded 1942-1943 residuals of only $6 "$. A further calculation of the same type gave a second correction $\Delta n_{2}=-0.000000014$.

We were thus able to obtain an orbit linking the two apparitions and considering a total of 128 observations ( 35 in 1867 and 93 in 1942-1943). The maximum residuals (in right ascension) do not exceed $1^{\prime \prime}$ in 1942-1943 and reach $2^{\prime \prime}$ in 1867 . The final elements were:

$$
\begin{aligned}
& \text { Epoch }=1942 \text { Oct. } 18.0 \\
& T=1942 \text { Dec. } 19.07104 \varphi=59.41721 \\
& M_{0}=358.42478 \quad n=91 " 35711 \\
& \omega=358.26892) \quad q=1.59534 \mathrm{AU} \\
& \Omega=78.59958\} \quad 1950.0 \quad Q=21.34181 \mathrm{AU} \\
& i=17.89615 \quad P=38.83872 \mathrm{yr} \text {. }
\end{aligned}
$$

These elements are not greatly different from those obtained from the observations in 1942-1943 alone.

It is of interest to integrate the above orbit over the 400-yr interval 1660-2060 in order to find approaches to the major planets and generally to investigate the evolution of the comet's orbit. During this period P/Stephan-Oterma had approaches to the Earth, Jupiter, Saturn, and Uranus; they are listed in Table I. At its next return $(T=1980$ Dec. 5.165) the comet will have rather a close approach to the Earth, minimum distance occurring 4 days after perihelion passage. As a result, conditions for observing the comet will be favourable.

The elements of the orbit have changed rather insignificantly during the 400-yr interval, the values at the beginning and end of the interval being given in Table II. Inside the interval the largest changes were as follows:

$$
\begin{aligned}
& 36.9 \leq P \leq 38.9 \\
& 91 " 4 \leq n \leq 96.1 \\
& 20.6 \leq Q \leq 21.3 .
\end{aligned}
$$




\section{TABLE I}

Minimum distances (in $\mathrm{AU}$ ) between $\mathbf{P} /$ Stephan-Oterma and the major planets

\begin{tabular}{lllll}
\hline & Earth & Jupiter & Saturn & Uranus \\
\hline 1720 May 12 & - & 1.954 & - & - \\
1731 Dec. 15 & - & - & - & 1.270 \\
1819 Aug. 30 & - & - & - & 2.171 \\
1866 Nov. 6 & 0.919 & - & - & - \\
1901 Apr. 14 & - & - & 2.848 & - \\
1903 Jan. 17 & - & 1.661 & - & - \\
1942 Dec. 7 & 0.631 & - & - & - \\
1980 Dec. 9 & 0.593 & - & - & - \\
1984 June 1 & - & - & 1.424 & - \\
\hline
\end{tabular}

TABLE II

Approximate elements at beginning and end of integration interval

\begin{tabular}{cclllllll}
\hline Date & $\omega$ & $\Omega$ & $i$ & $e$ & $P(\mathrm{yr})$ & $q(\mathrm{AU})$ & $Q(\mathrm{AU})$ & $n$ \\
\hline 1660 & $357^{\circ}$ & $81^{\circ}$ & $18^{\circ}$ & 0.858 & 38.07 & 1.60 & 21.03 & $93 \prime \prime 19$ \\
2060 & 0 & 77 & 18 & 0.860 & 38.42 & 1.59 & 21.18 & 92.36
\end{tabular}

All the calculations were made on the BESM-4 computer using programmes prepared by the staff of the Institute for Theoretical Astronomy: N. A. Belyaev, N. A. Bokhan, S. G. Makover and the author.

\section{References}

Becker, L.: 1891, Monthly Notices Roy. Astron. Soc. 51, 475.

Dubyago, A. D.: 1943, Astron. Tsirk. No. 17. 